\title{
Hot Spot Phenomenon and Greedy Routing Failure in Mobile Ad-hoc Networks
}

\author{
Ahmad Khader Habboush ${ }^{1}$ \\ ${ }^{1}$ Department of computer seines and information technology, Jerash University, Jordan \\ Correspondence: Ahmad Khader Habboush, Department of computer seines and information technology, Jerash \\ University, Jordan. E-mail: ahmad_ram2001@yahoo.com
}

Received: January 22, 2019

Accepted: February 8, 2019

Online Published: March 31, 2019

doi:10.5539/mas.v13n4p89

URL: https://doi.org/10.5539/mas.v13n4p89

\begin{abstract}
Lately, Greedy Forwarding Strategy (GFS) is express as a basic mechanistic in geographic routing for Mobile Ad Hoc Networks (MANETs). GFS employs the shortest path between two connected nodes. The research's analysis of networks that using GFS indicates that some areas of the MANET are more decumbent to high traffic load compared with other places. This situation is called hot spot phenomenon. Accordingly, these hot spot nodes become constrained in terms of many issues and seem to be died fast. The performance of GFS becomes limited to these hot spot nodes. The whole MANET fails, as GFS fails to function well in this area. This paper takes an in depth look at the reason behind greedy failure. Lastly, the work concludes the most appropriate characteristics that should be consider for enhancing greedy scheme to be compatible with the unique features of MANET.
\end{abstract}

Keywords: GFS, MANET, geographic routing, shortest path

\section{Introduction}

The Mobile Ad Hoc Network (MANET) also known as ad hoc wireless network appeared in the early 1970s [1]. It cause to grow and become more mature to be used in different applications such as battlefield and, disaster area. The Mobile Ad hoc Networks consists of a group of wireless mobile nodes such as personal digital assistance, Digital Audio Player, Mobile phone, laptop, etc. [2]. Those wireless mobile nodes have the ability to send or receive at the same time and can act as router. In Mobile Ad hoc Networks, the communication between two wireless mobile nodes achieved through several number of hops that shapes the route between them. In Mobile Ad Hoc Networks, participating nodes are mobile thus; they can join or leave the network at any time they wish. As results of that, Mobile Ad Hoc Networks have new features that are totally different from traditional networks, such as robustness, rapid deployment, flexible-topologies and rapid deployment [3].

Based on the new features of MANETs, the routing protocols have new challenges to guarantee high performance. Those issues such as; limited bandwidth, link disconnected because of node mobility, limited power of wireless mobile nodes. Because of the new challenges, routing algorithms in Mobile ad hoc networks in need to solve those issues to achieve the desired performance in MANET [4].

One of the most popular algorithms that used in MANETs is greedy forwarding strategy (GFS) which is deemed the essential algorithm in the geographic routing protocols. The routing decision when using GFS is made by using the location information of participating nodes and the distance between mobile nodes. But, in deed the GFS is unable to solve the new issues in MANET. Therefore, GFS may cannot guarantee succeed with high performance. GFS failure achieved because of using geometric calculation as the only routing criterion [5].

This paper introduces a complete study of the problems behind GFS failure. The rest of this paper is organized as follows. The next three Sections introduce a quick review of the effects of nodes' condition, their mobility attributes, and inaccuracy of position information on greedy functionality. Section IV provides some recommendations and finally, in Section IIV we introduce conclusion and future work.

\section{Hot Spot Phenomenon and Greedy Failure}

The current greedy algorithm considers the shortest path as a measure of route cost to make routing decisions [4]. Figure 1, shows the case of greedy forwarding approach during establishing the rout between the mobile nodes. The source node $S$ has data to be forwarded to the destination node $D$. Node $S$ has a group of neighbours, $E$, $H, M$, and $R$ in the direction of the target. One function of the node applies GFS is to compare distance between 
neighbours, $E, H, M$, and $R$, and the destination node $D$ and decides the closest neighbor to the destination. According to the distance condition (shortest path), node $E$ is the closest node to the destination $D$. Hence, node $S$ selects $E$ as the optimal next-rely node amongst other neigbours.

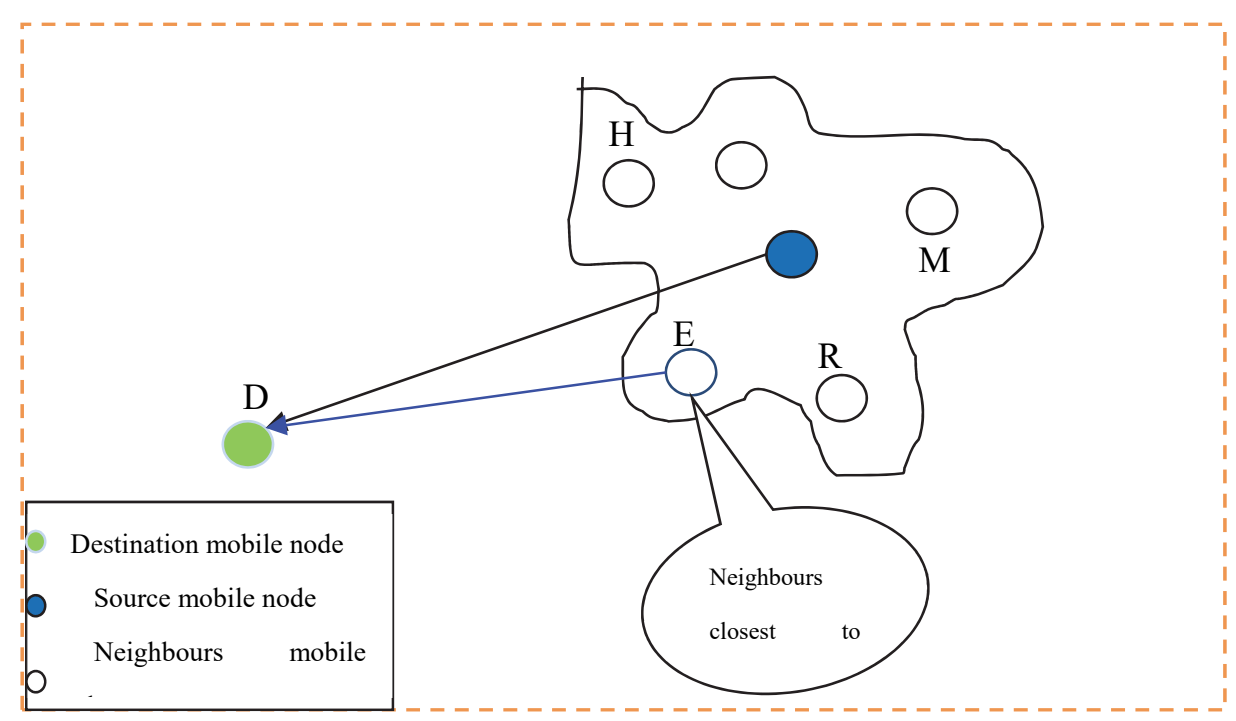

Figure 1. Greedy Routing in Mobile Ad Hoc Network

Theoretically, distance metric as deciding factor is easy to implement and reliable in dynamic environments [5]. Through implementation phase, this selection process is unreliable, because the proper operation of this function can be affected by very serious and important factors which are not considered. These factors mainly revolved around the conditions of participating nodes, their mobility attributes, and the accuracy of their position information.

\section{The Effects of Participating Nodes' Conditions}

Greedy forwarding strategy utilizes the shortest path between two communicating nodes. The shortest path between a pair of nodes represents the straight line between them as it depicted in Figure 2. Routing protocols based on the shortest path cannot fairly distribute the routing load among mobile nodes [4]. Hence, use shortest path results in network hot-spots. In other words, some areas of the MANET are more prone to high traffic load.

Accordingly, this exhausted area of MANET will have the most congested nodes, because those nodes required to forward packets more often than others [6]. Also, those nodes will have more use of battery power. Thus, their lifetime will be decreased very fast. Adding, as those nodes die because they come out of energy or nodes suddenly disappear from the MANET, this will vary MANET connectivity. Therefore, a node degree will change frequently [7].

Current greedy forwarding strategy does not consider the conditions of the next relay node in its selection process. Consequently, a shortest path sometimes incurs a higher packet loss, higher end-to-end delay, and least packet delivery ratio than some other available routes [8]. 


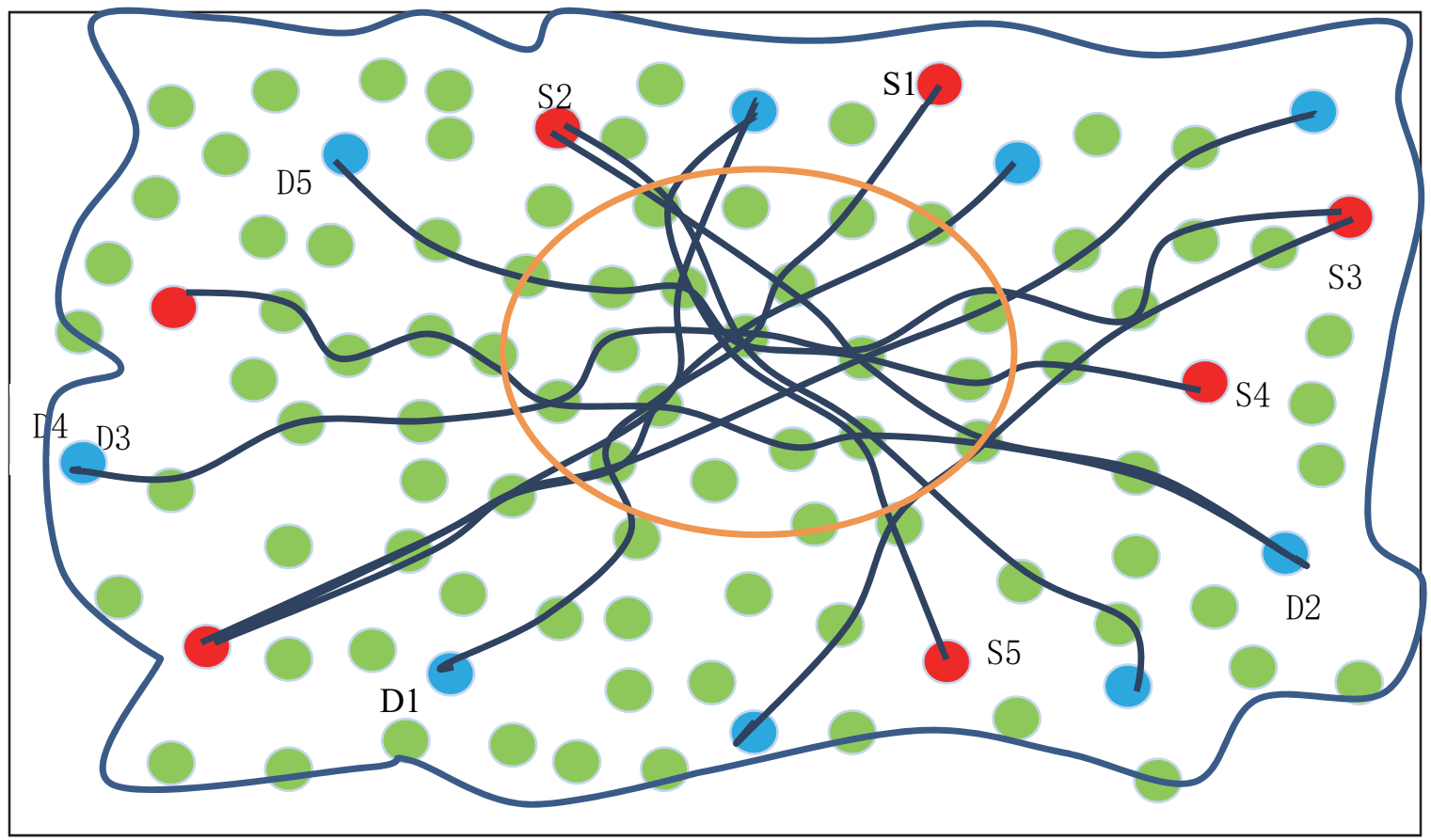

Figure 2. The high traffic load area problem

\section{The Effects of Mobility Attributes}

Mobility in MANET is an essential part of the network architecture block. Hence it should be taken into account when designing the routing protocol. Mobility in MANET have some negative effects, it can cause frequent unpredictable topology changes [9]. Another consequence of mobility is the increased rate of link break, and creates new links, which increases the volume of control traffic that required establishing and maintaining the routes.

Mobility metrics affect the performance of routing protocols in MANET. Among these metrics as depicted in Figure 3, speed, motion direction, link stability, and beacon interval time, as classified in [10]. With the greedy algorithm, next relay node mobility metrics were not considering for making selection decision. Selected node may have an opposite movement direction, and different level of speed (higher or slower) with respect to source node or forwarder node, which vary the link life time between two communicated nodes.

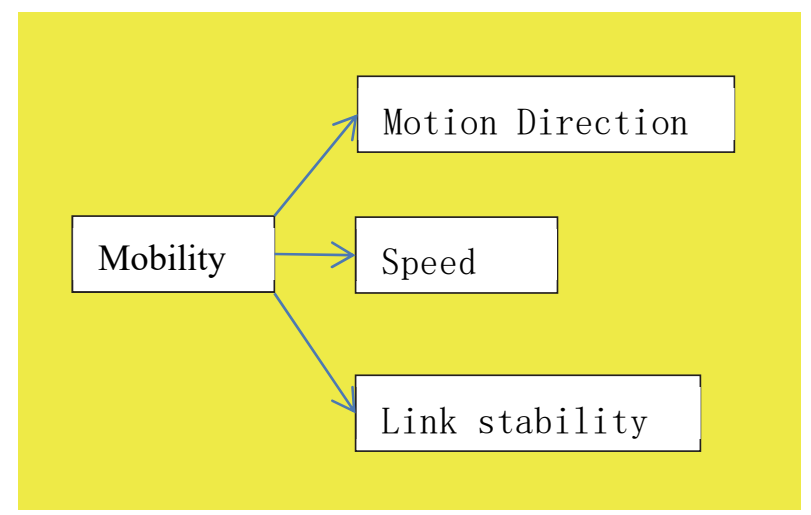

Figure 3. Mobility metrics

\section{The Effects of Position Inaccuracy of Participating Nodes}

Due to its efficiency and scalability, Greedy algorithm used as basic mode of operation for most geographic routing protocols for MANET. All proposed geographic routing protocols assumed the availability of accurate location 
information [11]. Meanwhile, this assumption is invalid in any realistic deployment because location measurement is often noisy and incurs some error.

Recently several studies on MANET [12,13] have shown that nodes position rarely to be accurate in such a high dynamic environment. Hence, always there is certain imprecise location information. Location errors can significantly impact the performance of the position-based routing protocols. Consequently, one of the drawbacks of the current greedy approach is that the position of the participating nodes needs to be known with an accuracy of a one-hop transmission range.

Inaccuracy of the position information in MANET refers to two main reasons; the first one due to GPS information nature, this reason will be beyond this work concern. The second reason (as discussed earlier) refers to node mobility, beacon packet interval time, and neighbour's entry expire-time in neighbours list.

Arbitrarily motion of MANETs' nodes causes unpredictable changes in network topology. Consequently, local topology changes frequently and rarely remains static. Thus, there is a need to update the changes that occur to node position due to nodes' mobility to all other neighbours [14,15]. Using a beacon messages mechanism solve this problem, but still accompanies with a main drawback in determining the optimal beacon interval in MANET environment. Adding, the validity of the neighbours' entries in a node's neighbours list is much related to the used updating mechanism and to the period of waiting time before a node removes neighbour's entry from its neighbours list.

\section{Summary of the Potential Problems and Recommendation}

Owing to the above problem, this section, introduces a quick summary of the potential harms that may occur due to using distance as the only deciding factor in GFS routing protocol. Ignorance of conditions of participating nodes, their mobility attributes, and the accuracy of their position information can have devastating consequences and degrade the performance of GFS. A shortest path sometimes incurs a higher packet loss, higher end-to-end delay, and least packet delivery ratio, decrease the lifetime of the network, the appearance of a local maximum problem (non-optimal path) and loop problem than some other available routes. Table 1, introduces a full information of each problem and the harmless cropped due to ignorance of each metric.

Table 1. Shortest path problem and recommended metrics

\begin{tabular}{|c|c|c|}
\hline \multicolumn{3}{|c|}{ Mobility attributes } \\
\hline Problem/Metrics & Consequences & Recommended Metrics \\
\hline Movement Direction & High packet loss, high end to end & Relative Direction \\
\hline Speed & delay, decrease packet delivery, & Relative Speed \\
\hline Link Life Time & non-optimal path, loop problem & Link Stability \\
\hline Beacon Packet Interval Time & & Dynamic Beaconing \\
\hline Neighbours List Entry lifetime & & Dynamic Entry Remover \\
\hline & $\begin{array}{l}\text { Position Inaccuracy } \\
\text { (n) }\end{array}$ & \\
\hline $\begin{array}{l}\text { Neighbours Inaccuracy } \\
\text { Destination Inaccuracy }\end{array}$ & $\begin{array}{l}\text { High packet loss, high end to end } \\
\text { delay, decrease packet delivery, } \\
\text { non-optimal path, loop problem. }\end{array}$ & $\begin{array}{l}\text { Dynamic Update } \\
\text { Dynamic Update }\end{array}$ \\
\hline
\end{tabular}

\section{Conclusion and Future Work}

Mobile ad hoc network, which run by wireless mobile devices, are in highest demand. The use of such network can be easily extended to places which cannot be wired, thus, it enhance a new types of daily life implementation, such as rescue operation. Recently, position-aware routing protocols have been given a special interested due to its several benefits against position-unaware routing protocols.

In this paper, the functionality of GFS has been deeply discussed. The reasons behind greedy failure have also been modeled and explained in depth. Each of which can severely degrade the performance of GFS in terms of MANET life-time, local maximum, packet delivery ratio, end-to-end delay, non-optimal route, and routing loop.

At the end, opportunities which could lead to further improvements have been identified to give the GFS the ability to be as a standalone routing technique without the aid of external supports. Presently, the efforts are directed to perform simulation experiments to show the severe consequences of greedy failure on the network performance in terms of the intended metrics. Furthermore, an enhancement for GFS protocol is in the high vision to be accomplished to improve its performance in the context of MANET. 


\section{References}

Aditya, R. S., Pai, M., Boussedjra, M., \& Mouzna, J. (2008). GPSR-L: Greedy Perimeter Stateless Routing with Lifetime for VANETS. In Proceedings of 8th International Conference on Intelligent Transport System Telecommunications, 2008, pp. 299-304.

Al-Akaidi, M., \& Alchaita, M. (2007). Link Stability and Mobility in Ad Hoc Wireless Networks. IET Communications, 1, 173-178. https://doi.org/10.1049/iet-com:20060273

Bansal, M. et al. (1999). Mobile Ad Hoc Networking (MANET): Routing Protocol Performance Issues and Evaluation Considerations. 1999. https://doi.org/10.17487/RFC2501

Boukerche, et al. (2011). Routing Protocols in Ad Hoc Networks: A Survey. Computer Networks (Elsevier), 55(13), 3032-3080, September 2011. https://doi.org//10.1016/j.comnet.2011.05.010

Cheng, R. E. I. H., \& Huang, C. (2012). Efficient Prediction-Based Location Updating and Destination Searching Mechanisms for Geographic Routing in Mobile Ad Hoc Networks. Journal of Information Science and Engineering, 28, 115-129, 2012. https://doi.org/10.2197/ipsjjip.26.132

D. Son, et al. (2004). The Effect of Mobility-Induced Location Errors on Geographic Routing in Mobile Ad Hoc Sensor Networks: Analysis and Improvement Using Mobility Prediction. Mobile Computing, IEEE Transactions on, 3, 233-245.

Finn, G. (1987). Routing and Addressing Problems in Large Metropolitan-Scale Internetworks," USC/Information Sciences Institute, Technical Report ISI/RR-87-180, March 1987. ERIC Number: ED290427

Karp, B., \& Kung, H. T. (2000). "GPSR: Greedy Perimeter Stateless Routing for Wireless Networks," in the Proceedings of the sixth annual international conference on Mobile computing and networking ACM Boston, MA, USA, 2000, pp. 243-254. https://doi.org/10.1.1.112.2772

L. Moraru, et al. (2008). Path Quality Detection Algorithms for Near Optimal Geographic Routing in Sensor Networks with Obstacles Wireless Communications and Mobile Computing. Wireless Communications and Mobile Computing, 10, 647-661, 2008. https://doi.org/10.1002/wcm.727

Marina, M., \& Das, S. (2005). Routing in Mobile Ad Hoc Networks. in Ad Hoc Networks, Springer, pp. 63-90, 2005.

Persia, S. et al. (2008). Impact of Mobility on Geographic Routing," In the Proceedings of 3rd International Symposium on Wireless Pervasive Computing, ISWPC 08., pp. 717-721.

Rührup, S. (2006). Position-based Routing Strategies. Ph.D Thesis, Paderborn, Germany.

T. Reddy, et al. (2006). Quality of Service Provisioning in Ad Hoc Wireless Networks: A Survey of Issues and Solutions. Ad Hoc Networks, 4, 83-124. https://doi.org/10.1016/j.adhoc.2004.r04.008

Venkatasubramanian, S., \& Gopala, N. (2010). A Quality of Service Architecture for Resource Provisioning and Rate Control in Mobile Ad Hoc Networks," International Journal of Ad hoc, Sensor \& Ubiquitous Computing (IJASUC), 1, 106-120. https://doi.org/10.5121/ijasuc.2010.1309

Xue, F., \& Kumar, P. R. (2004). The Number of Neighbors Needed for Connectivity of Wireless Networks. Wireless Networks, 10, 169-181, 2004. https://doi.org/10.1023/B:WINE.0000013081.09837.c0

\section{Copyrights}

Copyright for this article is retained by the author(s), with first publication rights granted to the journal.

This is an open-access article distributed under the terms and conditions of the Creative Commons Attribution license (http://creativecommons.org/licenses/by/4.0/). 